\title{
Reduction of the Velocity Impact on the Magnetic Flux Leakage Signal
}

\author{
Zbigniew Usarek $^{1}$ (1) $\cdot$ Marek Chmielewski ${ }^{1} \cdot$ Leszek Piotrowski $^{1}$
}

Received: 27 September 2018 / Accepted: 18 February 2019 / Published online: 26 February 2019

(c) The Author(s) 2019

\begin{abstract}
The velocity effect on the magnetic flux leakage (MFL) signal was investigated in this paper. Experiments were performed for velocity of the MFL tool within the range of $0-2 \mathrm{~m} / \mathrm{s}$. The velocity was not constant during each measurement to imitate real operational conditions of the MFL tool. Two components of the leakage were measured, i.e. the tangential to the motion direction $(x)$ and the normal to the investigated surface $(z)$. In addition to them, the gradient of the normal component in $x$ direction was measured with the use of two adjacent sensors. The normal component was found to be the most sensitive to the velocity effect. It was shown that the baseline value of the normal component is proportional to the velocity. An empirical compensation scheme was formulated, and it was used to minimize distortions of the normal component caused by the velocity effect. The finite element method was used to study the distribution of velocity-induced eddy currents in the investigated plate. It was stated that eddy currents generated below the poles lead to a change of plate magnetization as well as to a change of magnetic field distribution above the top surface of the plate, what is observed as a shift of the MFL signal baselines.
\end{abstract}

Keywords Magnetic flux leakage $\cdot$ Velocity effect $\cdot$ Eddy current $\cdot$ Finite element method

\section{Introduction}

Magnetic flux leakage (MFL) is commonly used as a qualitative inspection method. Modern MFL tools are suitable for fast detection and classification of magnetic anomalies. Some of these anomalies are indicators of macroscopic defects, such as corrosion pits, dents, cracks etc. Estimation of their dimensions is a non-trivial issue while considering number of factors that influence the magnitude of an MFL signal. Further development of MFL requires efforts to minimize influence of various factors on the MFL signal. One of the factors that strongly affects the MFL signal is velocity of an MFL tool, which is of special interest in high speed rail inspection [1] and pipeline pigging [2]. In the latter case velocity of the tool can exceed $10 \mathrm{~m} / \mathrm{s}$ [3-5], while velocity ranges of $0.5-4 \mathrm{~m} / \mathrm{s}$ are recommended for mapping corrosion in pipelines. But even for so relatively low values of the velocity, its impact on the MFL signal cannot be neglected. According to Faraday's law of induction, the electromotive

Zbigniew Usarek

zbigniew.usarek@pg.edu.pl

1 Department of Solid State Physics, Faculty of Applied Physics and Mathematics, Gdansk University of Technology, Gabriela Narutowicza Street 11/12, 80-233 Gdansk, Poland force generates eddy currents within conductive material if its velocity is non-parallel to the direction of a static magnetic field:

$\overrightarrow{J_{e}}=\sigma \vec{v} \times \vec{B}$

where $\boldsymbol{J}_{\boldsymbol{e}}$-eddy current density, $\sigma$-electrical conductivity, $\boldsymbol{v}$-velocity, $\boldsymbol{B}$-magnetic flux density.

Equation (1) indicates that eddy current density reaches the maximum when $\boldsymbol{B}$ is perpendicular to $\boldsymbol{v}$. In the case of a yoke magnetizer travelling above a surface of a steel object, eddy current is generated mainly under the magnetizer poles. Eddy current generated below the poles reduces the magnetic flux density in the steel object in accordance to Lenz's law [3, 4]. Furthermore, initially symmetric distribution of $\boldsymbol{B}$ inside the object becomes asymmetric as the velocity rises $[3,6]$. The area of the highest magnetization migrates towards the back positioned pole of the magnetizer [4]. Some of components of the MFL signal are also modified due to the velocity. Baselines of this components as well as amplitudes of detected anomalies are in general nonlinearly dependent on the velocity $[1,7,8]$. The anomalies of the MFL signal carry information about shapes of defects e.g. metal losses. However, as reported in $[4,5,9,10]$, a profile of the signal anomaly becomes more distorted (usually more asymmetric) as the velocity increases. All these effects hinder evaluation of defect dimensions and its shape 
as well. One can define two different approaches to solve this problem. First approach involves optimization of an MFL tool in order to minimize the velocity influence on the MFL signal [11]. Second approach is based on post-processing and transformation of the signal to its stationary form.

Only few studies among those devoted to the issue of velocity-induced eddy current include a proposition of restoration of the MFL signal to its stationary form [5, 12-14]. In his thesis [12] Mandayam presented a method of MFL signal processing that lead to a velocity invariant MFL signal. He listed two classes of compensation schemes. A scheme of first class requires the velocity value as input data to perform the compensation of the signal. A scheme of second class does not require such data and this kind of the scheme was used by Mandayam to obtain the velocity invariant MFL signal. In this case, an unsupervised learning algorithm was used to determine coefficients of the restoration filter. Lei et al. [13] used a learning algorithm, which was based on radial basis function neural network, to compensate velocity effect on the MFL signal. Accuracy of those and similar compensation schemes depends on quantity and quality of input training data. Computer simulations can provide large amount of training data. Experiments are more timeconsuming and expensive in this case but a compensation scheme based on experimental data is usually more robust than simulation-based one. In comparison to aforementioned compensation schemes one proposed by Park and Park [5] is much simpler. For a particular class of MFL signal anomalies they performed an analysis of various anomaly parameters such as a peak-to-peak value of an anomaly as a function of the velocity. So obtained relations were used to restore the stationary form of the MFL signal. Drawback of this approach is limited application of so formulated compensation scheme to the specific class of MFL signal anomalies.

Most studies presented in literature of the subject include a small number of experimental results. Therefore, in this study MFL signal dependence on the velocity was investigated using experimental data. A range of the velocity was selected so as to fill the data gap existing for relatively low velocities, i.e. up to $2 \mathrm{~m} / \mathrm{s}$. Presented study was focused on signal baseline dependence on the velocity of the MFL tool.

\section{Experimental Setup}

The MFL tool used in the experiment is presented in Fig. 1. It comprised three magnetizers with two wheels each, a digital encoder, and a measurement module. A single magnetizer consisted of two neodymium magnets connected together using a steel beam. The digital encoder enabled to determine displacement of the MFL tool, as well as its velocity.

The measurement module, as presented in Fig. 2, comprised of ten channels for each signal component. Each

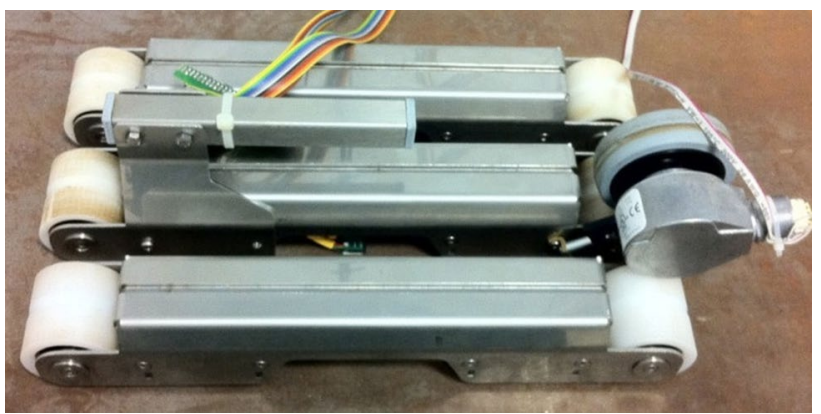

Fig. 1 The MFL tool used in the experiment

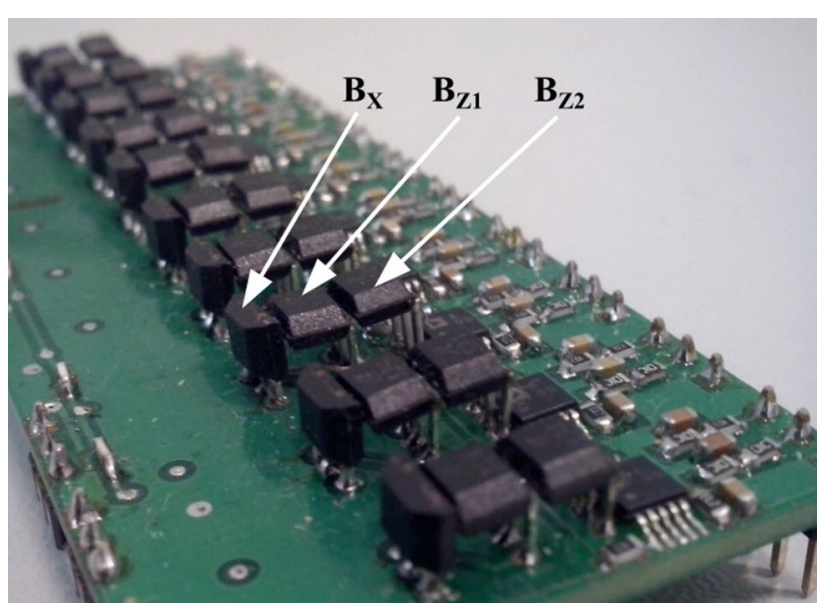

Fig. 2 The measurement module. Three sensors arranged side by side were used to measure three kinds of a signal: $B_{x}, B_{z 1},\left(B_{z 1}-B_{z 2}\right)$

channel is responsible for scanning adjacent measurement lines parallel to $x$ axis. Therefore, the measurement module in general enables to obtain a $2 \mathrm{D}$ distribution of the MFL. For the sake of simplicity in this work only results from the channel 5 (according to Fig. 2 the fifth one from the front) were presented.

The location of the measurement module is schematically depicted in Fig. 3. Linear Hall effect sensors A1324 were used to measure magnetic flux leakage. Three voltage signals were measured: $B_{x}$ sensor output, $B_{z l}$ sensor output, and difference of $B_{z 1}$ and $B_{z 2}$ outputs. An output of a $B_{x}$ sensor was directly proportional to the tangential (horizontal) component of MFL while an output of a $B_{z I}$ sensor was directly proportional to the normal (vertical) component of MFL (hereafter referred to as $B_{z}$ ). A differential signal $\left(B_{z 1}-B_{z 2}\right)$, that was proportional to slope of $B_{z}$ distribution measured along the $x$ direction, is hereafter referred to as the gradient, $\partial B_{z} / \partial x$.

The MFL tool was used to scan a test plate made of S355 steel grade. Four artificial defects were milled on one of plate surfaces. The defects had the form of slots of 


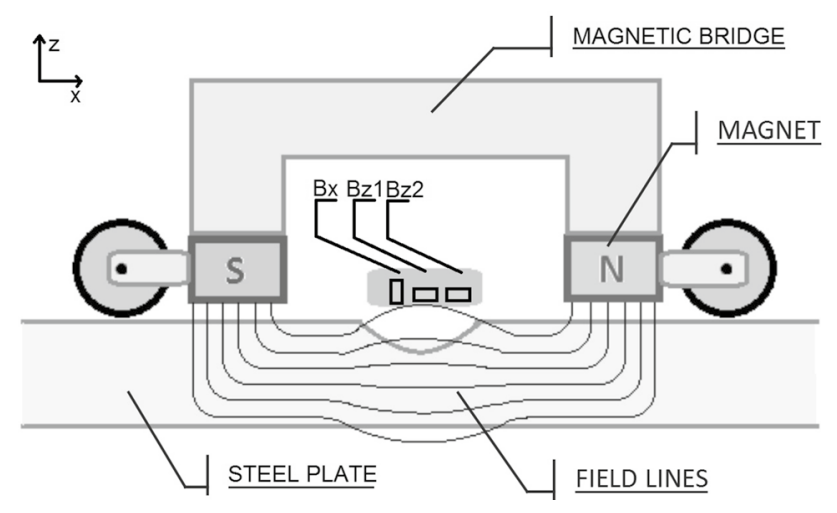

Fig. 3 A schematic of the MFL tool with the indicated location of the sensors
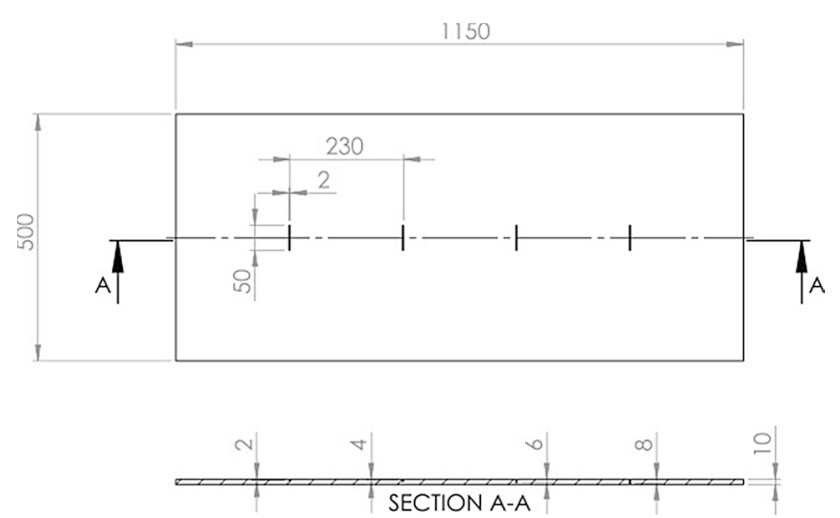

Fig. 4 Dimensions of the test plate and manufactured artificial defects

different depth. Dimensions of the plate and the defects are presented in Fig. 4.

The MFL tool was moved with the use of manual drive. Its displacement was parallel to a plate symmetry plane that is coplanar with the section A. Although signals coming from ten channels were recorded, only those from the channel nearest to the plate center are presented in this study.

\section{Results and Discussion}

\subsection{Experimental Results}

A series of measurements was performed for the steel plate with the rectangular slots. The velocity of the MFL tool was not constant during measurements as in the case of an inline pipeline inspection. Figure 5 shows a comparison of MFL signals obtained for a measurement made with a low velocity (below $0.3 \mathrm{~m} / \mathrm{s}$ ) and a measurement made with the target velocity of $1.5 \mathrm{~m} / \mathrm{s}$. As it can be seen in Fig. 5, each component of the measured signal has a different vulnerability to the velocity variation. Locations of four rectangular defects

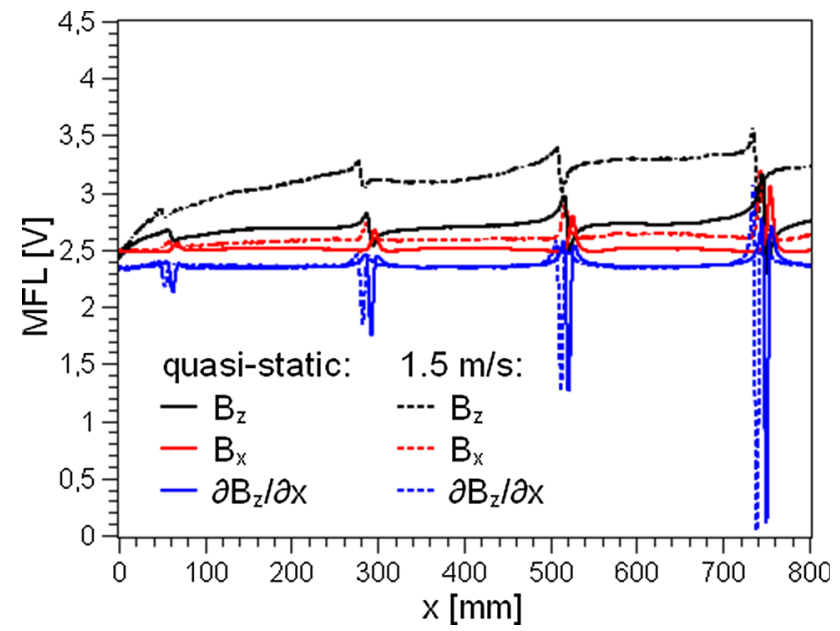

Fig. 5 Comparison of the MFL signal components measured in a quasi-static manner and with the target velocity of $1.5 \mathrm{~m} / \mathrm{s}$

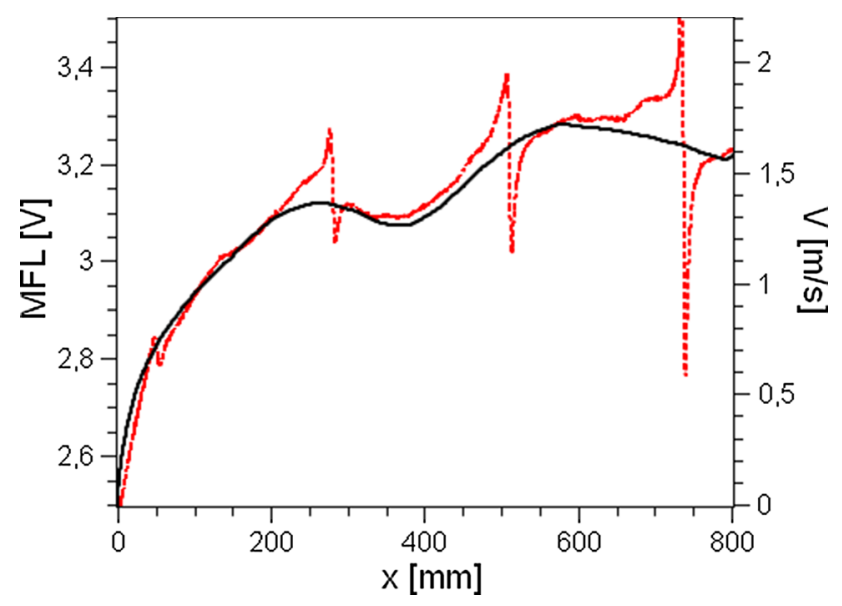

Fig. 6 Comparison of the MFL signal ( $B_{z}$ component) and the velocity of the MFL tool during the measurement

can be indicated on the basis of four signal anomalies. As the depth is the only geometric variable, it can be stated that peak-to-peak values of the anomalies increase with the increasing depth of a defect.

From the starting point $(x=0)$ the velocity was increased up to a predetermined value. For the measurement, which results are presented in Fig. 5, this value was approximately equal to $1.5 \mathrm{~m} / \mathrm{s}$. All measurements were characterized by a non-monotonic change of the velocity. Therefore, the influence of the variable velocity on the measured MFL signal was investigated. Figure 6 shows the variation of the velocity of the MFL tool during one of the measurements made for the investigated steel plate and the corresponding profile of $B_{z}$ component. This component is the most vulnerable to velocity changes. It is demonstrated by the change of the $B_{z}$ baseline which is qualitatively similar to the velocity profile. 
Therefore, the quantitative analysis of an anomaly distorted due to velocity changes is burdened with the velocity-related uncertainty.

\subsection{Compensation of the MFL Signal}

Dependencies of baselines of each signal component on the velocity were investigated. Three reference points located on the measuring path were selected as follows: $x_{1}=200 \mathrm{~mm}$, $x_{2}=400 \mathrm{~mm}, x_{3}=600 \mathrm{~mm}$. To minimize the impact of anomalies, locations of the reference points were selected as far as possible from the defects, i.e. at the midpoints of three virtual segments which connect neighboring defects. At these points, values of three signal components were extracted from the results of eight measurements performed with different target velocities. As a result, 24 values for each signal component was presented in Fig. 7 as a function of the velocity. Based on the results showed in Fig. 7 one can confirm that the velocity impact is the highest for the normal component, $B_{z}$. The relation between $B_{z}$ and the velocity value, $v$, can be approximated with the use of a linear function. Application of the least-square regression leads to the following formula: $\mathrm{MFL}_{-} \mathrm{B}_{\mathrm{z}}(v)=0.446 v+2.494$. The goodness of fit was evaluated by calculating the Chi squared value $\chi^{2}=0.0078$ and the corresponding coefficient of determination $\mathrm{R}^{2}=0.994$, which means very good fit.

The tangential component, $B_{x}$, is less dependent on the velocity compared with $B_{z}$. Moreover, although $B_{x}$ also increases with the velocity, it does not manifest linear dependence. The gradient, $\partial B_{z} / \partial x$, is the least dependent on the velocity. The relation between $\partial B_{z} / \partial x$ and the velocity is non-monotonic and characterized by weak fluctuations. The most probable reason of these fluctuations is variable acceleration of the MFL tool. $B_{z}$ is an implicit function of

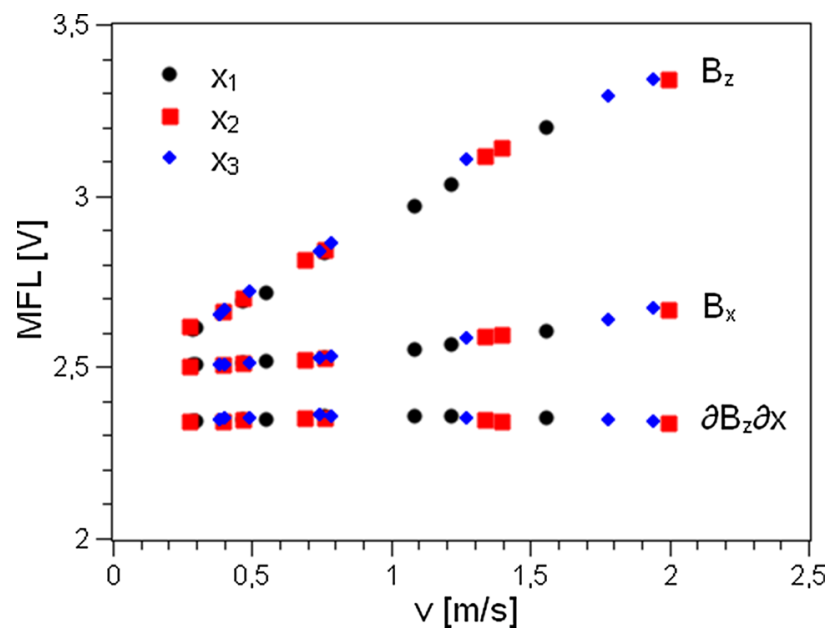

Fig. 7 Values of the MFL components as functions of the velocity for three reference points time, $B_{z}(x(t), v(t))$. As $B_{z}$ is approximately proportional to the velocity, $v(t)$, it can be deduced that its partial derivative is proportional to acceleration of the MFL tool, $\mathrm{d} v(t) / \mathrm{d} t$.

The profile of $B_{z}$ obtained for the fast measurement (characterized by the velocity profile presented in Fig. 5) was compensated using the formula: $\mathrm{MFL}_{-} \mathrm{B}_{\mathrm{z}}(x, v=0)=\mathrm{MFL}$ $\mathrm{B}_{\mathrm{z}}(x, v)-0.446 v$. The result of this compensation is presented in Fig. 8. The profile of $B_{z}$ obtained for a slower measurement (approximately $0.4 \mathrm{~m} / \mathrm{s}$ ) is also presented in Fig. 8 for comparison. As the velocity decreases, the baseline of MFL_ $B_{z}$ tends to the level of $2.5 \mathrm{~V}$, which corresponds to the static value of the MFL_B $B_{z}$ measured by the MFL tool in a non-defected region of the steel plate.

\subsection{Numerical Analysis}

Simulations using the finite element method (FEM) were performed to study physical foundations of the observed MFL signal dependence on the velocity. Software named Faraday from Integrated Engineering Software was used to perform the simulations. Geometry used for the simulations is depicted in Fig. 9. Three magnetic yokes above the surface of the steel plate were modelled. Geometry of the static model had two symmetry planes, $x z$ and $y z$. However, when the velocity in $x$ direction was non-zero, the $y z$ plane could not be treated as a symmetry plane. The magnetic insulation boundary condition was assigned to the $x z$ plane, which is a mirror symmetry plane for the magnetic field. This means that the magnetic field is zero in the normal direction to the boundary, whereas excitation currents (for modelling permanent magnets) are antisymmetric with respect to the boundary. There are four unique domains in the model that are numbered in Fig. 9. First domain represents a piece of

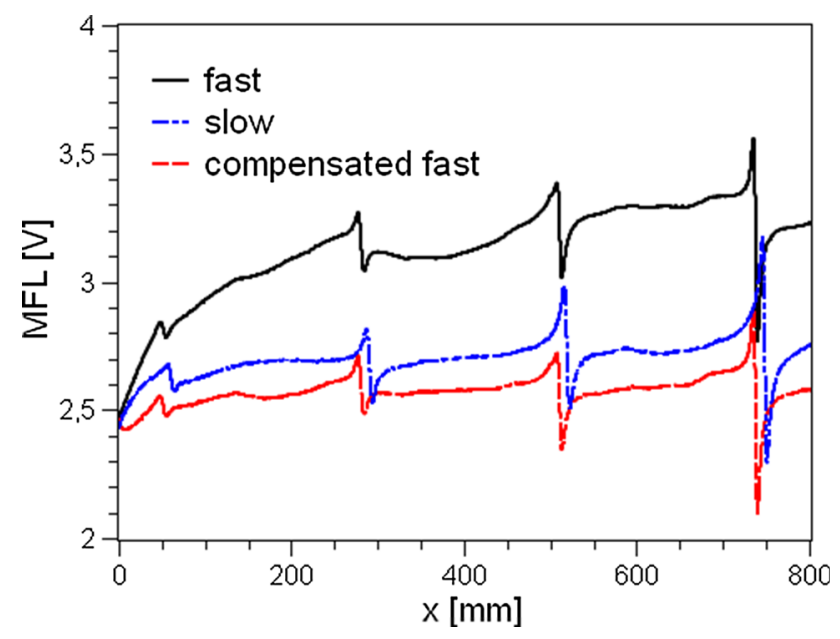

Fig. 8 Primary form of the $B_{z}$ profile for fast measurement and its compensated version. Results of a relatively slow measurement are also presented for comparison 


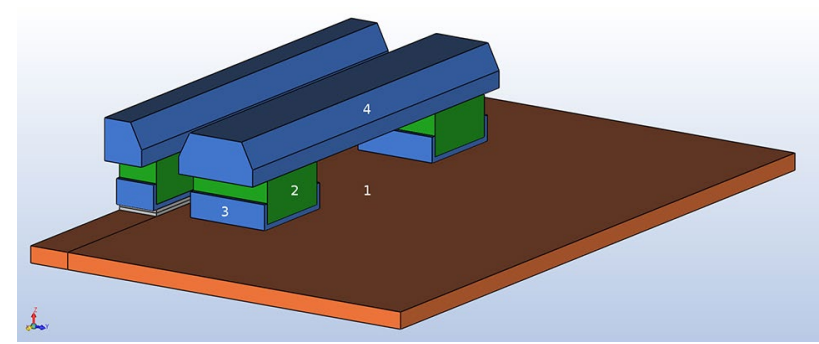

Fig. 9 Geometry used in the numerical study. Four numbered domains represent following components of the geometry: (1) steel plate, (2) permanent magnet, (3) protection of the magnet, (4) magnetic bridge

the steel plate. Its width ( $y$ dimension) is equal to the width of the real steel plate and its length was shortened so as not to affect the magnetic circuit significantly. Presence of $20 \%$ metal loss on the top surface of the plate was considered. Second domain comprises a neodymium permanent magnet. Third domain represents a C-shaped steel protection of the magnet. Fourth domain comprises a magnetic bridge connecting two magnets and thus closing the magnetic circuit.

Materials assigned to the domains presented in Fig. 9 had nonlinear magnetic properties. Initial magnetization curves, presented in Fig. 10, were measured experimentally for materials of domains 1,3 and $4 . B(H)$ curve of the plate material is depicted in Fig. 10a. As the domains 3 and 4 were made of the same material, they had the common $B(H)$ curve presented in Fig. 10b. Nonlinear magnetic properties were included in the model by implementing $B(H)$ curves in the table form into a material library of Faraday. Neodymium N45H magnet was assigned as the material of the second type of domain $\left(H_{c}=-1027 \mathrm{kA} / \mathrm{m}\right.$, $\left.B_{\mathrm{r}}=1.35 \mathrm{~T}\right)$. Domains made of magnetic materials were surrounded by the FEM rectangular box (air domain invisible in Fig. 9). Dimensions of the FEM box were 1.5 times longer than maximum dimensions of created geometry. On external surfaces of the FEM box, excluding $x z$ plane, the Dirichlet boundary condition was defined (vector magnetic potential $\boldsymbol{A}=0$ ).

Due to material nonlinearity, the problem was solved with the use of GMRES (Generalized Minimal Residual method) algorithm. The criteria of convergence was achieved when the residual was less than $\varepsilon=0.01$.

Relative velocity of the plate with reference to the magnetizer was set as $\boldsymbol{v}=-1 \boldsymbol{i}[\mathrm{m} / \mathrm{s}]$. As the material of the plate was assumed to be electrically conductive, eddy currents were created within the volume of the plate. Figure 11 shows a contour plot of surface current density in $x z$ plane. One can indicate three regions of eddy current generation: two below poles of the magnetizer and one in the vicinity of the metal loss. Eddy currents generated below the poles lead to a change of plate magnetization as well as to a change of magnetic field distribution above the top surface of the plate. In particular it results in a shift of measured components of magnetic field. Currents generated in the vicinity of the metal loss have the significant impact on the anomaly of MFL distribution. However, this impact was not considered in the presented study.

Figure 12 shows evolution of $B_{z}$ distribution in the region between the plate and the magnetizer when the velocity is increased from 0.01 (top panel) to $1 \mathrm{~m} / \mathrm{s}$ (bottom panel). Presented results refer to the plate without the metal loss. Initially symmetric distribution of $B_{z}$ becomes asymmetric when the velocity impact is non-negligible. It results in a change of a measured value of $B_{z}$ for a fixed location of a magnetic sensor. The sign of this change can be either negative or positive depending on the mutual orientation of the plate magnetization direction and the velocity. Therefore, one can state that eddy currents generated under the poles of the magnetizer were responsible for the experimentally observed shift of $B_{x}$ and $B_{z}$ baselines.

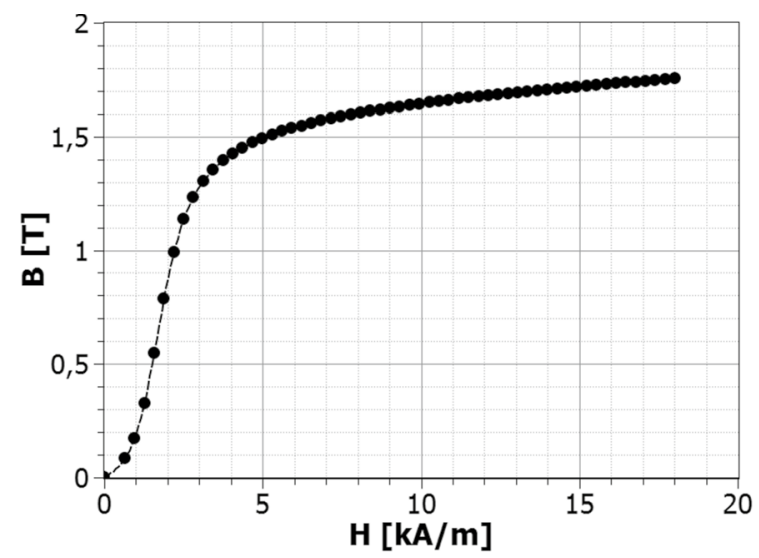

(a)

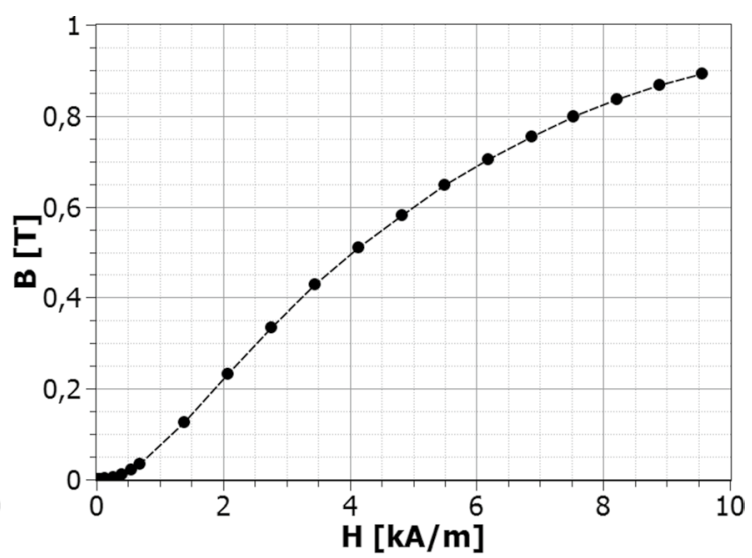

(b)

Fig. 10 Initial magnetization curves of: a steel plate, $\mathbf{b}$ protection of the magnet and magnetic bridge 


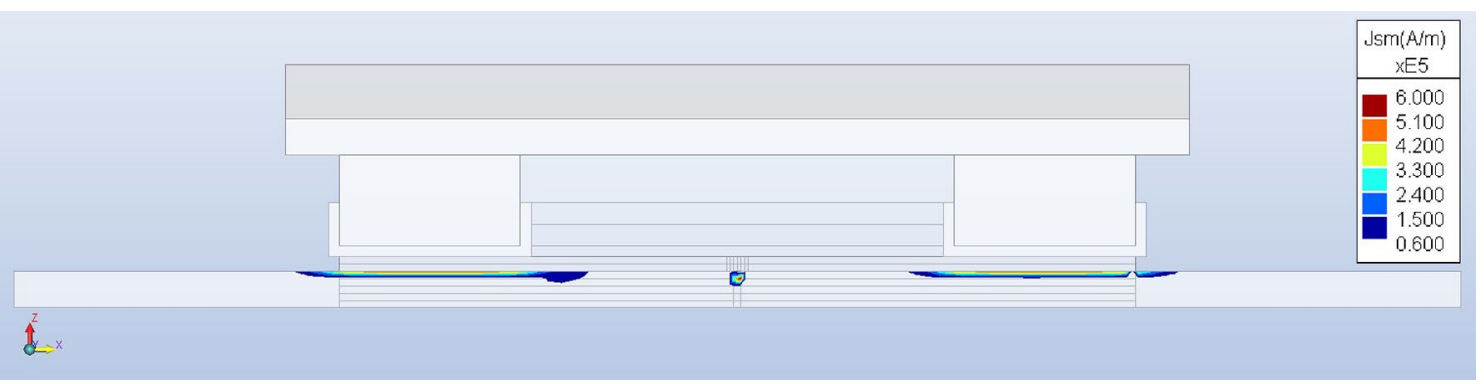

Fig. 11 Density of surface current induced due to motion of the magnetizer

Fig. 12 Distribution of $B_{z}$ between the plate and the magnetizer for different velocities. Results presented on the top and bottom panel refer to 0.01 and $1 \mathrm{~m} / \mathrm{s}$ respectively

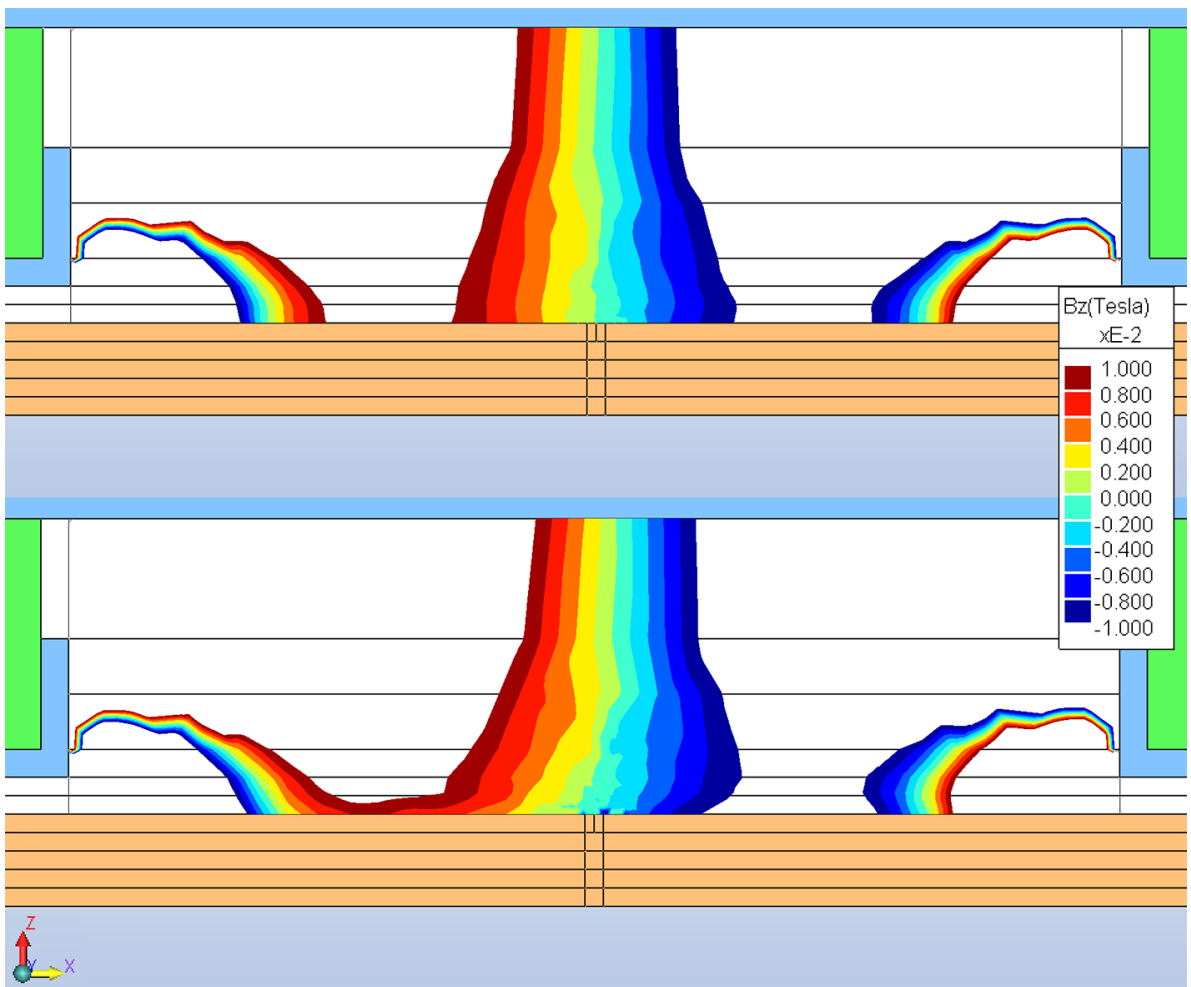

\section{Conclusions}

As shown, MFL signal distortions resulting from variable velocity of the magnetizer can be reduced using a relatively simple compensation scheme. At first, relations between measured components of the signal and the velocity were determined for this purpose. These relations are specific and dependent on many factors such as:

- Geometry and materials of an MFL tool,

- Position of sensors,

- Properties of a ferromagnetic object under investigation.

The component normal to the top surface of the steel plate, $B_{z}$, was recognized as the most sensitive to velocity changes in time. Proposed compensation scheme takes into account only the impact of eddy currents induced under the poles of the magnetizer. Reduction of the impact of eddy currents induced in the vicinity of a metal loss will be the subject of future research. Nonetheless, the results obtained with the use of the simplified compensation scheme suggest the possibility of reducing MFL signal distortions, when the velocity of the MFL tool is variable. Strong enough distortions of the MFL signal can be wrongly classified as fingerprints of defects. Proposed compensation scheme reduces probability of this kind of false positive indications. In the presented study, measured MFL signal were post-processed, but one can imagine that the compensation scheme could work online as well. Online compensation requires a sensor with offset terminals to stabilize the operating point of the sensor. 
Proportional to the velocity, properly scaled, and inversed voltage feeds the offset input. Such a kind of online compensation can protect the sensor against its saturation. Future work will be focused on implementation of abovementioned solutions to the MFL measuring system.

Acknowledgements This work was supported by the National Centre for Research and Development, Poland [project INNOTECH-K2/ IN2/53/182767/NCBR/12].

Open Access This article is distributed under the terms of the Creative Commons Attribution 4.0 International License (http://creativeco mmons.org/licenses/by/4.0/), which permits unrestricted use, distribution, and reproduction in any medium, provided you give appropriate credit to the original author(s) and the source, provide a link to the Creative Commons license, and indicate if changes were made.

\section{References}

1. Chen, Z., Xuan, J., Wang, P., Wang, H.: Simulation on high speed rail magnetic flux leakage inspection. In: Instrumentation and Measurement Technology Conference (I2MTC), 2011 IEEE. pp. $1-5(2011)$

2. Tan Tien Nguyen, Hui Ryong Yoo, Yong Woo Rho, Sang Bong Kim: Speed control of PIG using bypass flow in natural gas pipeline. In: ISIE 2001. 2001 IEEE International Symposium on Industrial Electronics Proceedings (Cat. No.01TH8570). pp. 863-868. IEEE (2001)

3. Nestleroth, J.B., Davis, R.J.: The effects of magnetizer velocity on magnetic flux leakage signals. In: Thompson, D.O., Chimenti, D.E. (eds.) Review of progress in quantitative nondestructive evaluation: volumes 12A and 12B, pp. 1891-1898. Springer, Boston (1993)
4. Katragadda, G., Sun, Y.S., Lord, W., Udpa, S.S., Udpa, L.: Velocity effects and their minimization in MFL inspection of pipelines-a numerical study. In: Thompson, D.O., Chimenti, D.E. (eds.) Review of progress in quantitative nondestructive evaluation, pp. 499-505. Plenum Press, New York (1995)

5. Park, G.S., Park, S.H.: Analysis of the velocity-induced eddy current in MFL type NDT. IEEE Trans. Magn. 40, 663-666 (2004)

6. Gan, Z., Chai, X.: Numerical simulation on magnetic flux leakage testing of the steel cable at different speed title. In: ICEOE 2011-2011 International Conference on Electronics and Optoelectronics, Proceedings. pp. 316-319 (2011)

7. Shin, Y.: Numerical prediction of operating conditions for $\mathrm{m}$;agnetic flux leakage inspection of moving steel sheet. IEEE Trans. Magn. 33, 2127-2130 (1997)

8. Wang, P., Gao, Y., Tian, G., Wang, H.: Velocity effect analysis of dynamic magnetization in high speed magnetic flux leakage inspection. NDT E Int. 64, 7-12 (2014)

9. Mandayam, S., Udpa, L., Udpa, S.S., Lord, W.: Invariance transformations for magnetic flux leakage signals. IEEE Trans. Magn. 32, 1577-1580 (1996)

10. Li, Y., Tian, G.Y., Ward, S.: Numerical simulation on magnetic flux leakage evaluation at high speed. NDT E Int. 39, 367-373 (2006)

11. Zhang, L., Belblidia, F., Cameron, I., Sienz, J., Boat, M., Pearson, N.: Influence of specimen velocity on the leakage signal in magnetic flux leakage type nondestructive testing. J. Nondestruct. Eval. 34, 6 (2015)

12. Mandayam, S.A.: Invariance transformations for processing NDE signals, https://lib.dr.iastate.edu/cgi/viewcontent.cgi?artic le $=12119 \&$ context $=$ rtd, (1996)

13. Lei, L., Wang, C., Ji, F., Wang, Q.: RBF-based compensation of velocity effects on MFL signals. Insight Non Destruct. Test. Cond. Monit. 51, 508-511 (2009)

14. Lu, S., Feng, J., Li, F., Liu, J.: Precise inversion for the reconstruction of arbitrary defect profiles considering velocity effect in magnetic flux leakage testing. IEEE Trans. Magn. 53, 1-12 (2017) 